\title{
Relazioni familiari al tempo del Covid-19 e buone prassi derivanti dal mondo dell'adozione
}

\author{
Alessia Tabacchi
}

\begin{abstract}
La pandemia da Covid-19 ha segnato la vita delle famiglie in modo significativo e dirompente. Da un lato, ha comportato una riorganizzazione dei ritmi di vita, in un precario gioco di equilibri nella conciliazione fra lavoro e famiglia. Dall'altro lato, ha chiesto inedite capacità nell'assunzione dell'incertezza quale orizzonte prossimo di ogni progettualità. Il contributo prende le mosse dall'analisi delle narrazioni di alcuni genitori adottivi al riguardo, al fine di precisare nessi significativi fra l'esperienza adottiva e l'emergenza sanitaria in corso. In prospettiva pedagogica, si tenta, in questa sede, di porre in luce alcune dimensioni utili allo sviluppo della resilienza familiare e dell'empowerment.
\end{abstract}

Parole chiave: adozione, relazioni familiari, Covid-19, emergenza, resilienza.

\begin{abstract}
The Covid-19 pandemic has marked families' lives in a significant and disruptive way. On the one hand, it involved a reorganization of the rhythms of life, in a precarious work and family balance. On the other hand, it asked for unprecedented skills in assuming uncertainty as the immediate horizon of any project. In this regard, the contribution starts from some thoughts that arose from the analysis of adoptive parents' narratives, to find significant links between the adoptive experience and the ongoing health emergency. From a pedagogical perspective, an attempt is here made to highlight some useful dimensions for the development of family resilience, and empowerment.
\end{abstract}

Keywords: adoption, family relationships, Covid-19, emergency, resilience.

\section{Il contesto di riferimento}

La pandemia da Covid-19 ha sconvolto l'ordinario procedere della vita familiare, con ripercussioni differenti in relazione alle realtà createsi

${ }^{1}$ Dottoranda in Scienze della persona e della formazione presso l'Università Cattolica del Sacro Cuore di Milano. 
all'interno dei singoli nuclei domestici. Si è parlato di famiglia «sospesa» (Centro di Ateneo Studi e Ricerche sulla famiglia, a cura di, 2020, passim), per indicare quanto implicato dall'emergenza sanitaria e dalla precarietà che con essa si accompagna. La rapida diffusione del virus ha minato la sicurezza personale e comunitaria, ha fatto emergere più che mai la vulnerabilità umana, ha chiesto sforzi e privazioni in vista del perseguimento del bene comune.

Le analisi condotte in questo periodo dai ricercatori di ambito interdisciplinare mettono in luce elementi di stress accanto a fattori di opportunità per le famiglie, così come processi di resilienza che hanno visto coinvolta a differente titolo la compagine familiare (Istat, 2020). La pedagogia della famiglia si interroga sulle situazioni di vita delle famiglie, considerando la pluralità delle realtà familiari e le specificità del contesto nel quale si trovano a vivere. Il sapere pedagogico trae importanti spunti di riflessione dalle narrazioni dei membri del sistema familiare. Come afferma Pati, «ogni famiglia è portatrice di un proprio universo di significati, di uno sguardo sul mondo e di un peculiare modo di vivere la vita e le relazioni» $(2017$, p. 25). All'interno del contributo, si intrecciano riflessioni che accostano lo studio delle famiglie originatesi mediante l'adozione all'odierna realtà emergenziale.

La pista di lavoro è scaturita dalla presenza come genitore adottivo all'interno di quattro incontri online organizzati da Afaiv (Associazione famiglie adottive insieme per la vita), un'associazione di famiglie adottive con sede in provincia di Varese. Il percorso si è svolto nel periodo fra aprile e giugno 2020, in orario serale. Tali eventi, condotti dalla Presidente dell'associazione e da una socia volontaria, hanno visto coinvolti genitori con figli adottivi di età compresa fra 0 e 10 anni, provenienti da percorsi di adozione nazionale e internazionale ${ }^{2}$.

Durante il primo incontro è stata data ai partecipanti la possibilità di raccontarsi, in particolare, con riferimento al periodo del lockdown e alle implicazioni per la propria famiglia. In seguito, le serate si sono focalizzate sul tema della narrazione e della costruzione dell'identità personale, con il coinvolgimento di differenti figure invitate a portare la propria testimonianza: una coppia di genitori con figli in età adolescenziale; due

${ }^{2}$ Ogni serata ha visto coinvolti un numero variabile di partecipanti (compreso fra le 25 e le 35 unità), in alcuni casi sono stati presenti entrambi i genitori, in altri solo un adulto per famiglia (in prevalenza la figura materna). (D'ora in poi, laddove non diversamente specificato, le note a piè di pagina si intendono a cura dell'Autrice, N.d.R.). 
ragazzi adottati; un'adulta adottata, formatrice sui temi della narrazione adottiva.

Il presente contributo non si sviluppa a partire da un previo progetto di ricerca. La partecipazione alle serate come osservatore partecipante e la pregnanza dei racconti e delle testimonianze ascoltate hanno sollecitato elementi di riflessività e hanno portato a cogliere notevoli nessi fra le implicazioni dell'emergenza sanitaria per le famiglie in oggetto e la passata esperienza adottiva. Si è pertanto scelto di porre l'accento su alcuni di questi aspetti, al fine di meglio comprendere le sfide poste dalla pandemia e le risorse per farvi fronte con protagonismo.

A livello metodologico, si è proceduto annotando i pensieri condivisi dai genitori adottivi. Le trascrizioni delle narrazioni sono state analizzate con metodo carta e matita. La comparazione dei contenuti ha permesso di individuare le parole, le tematiche e le strutture di significato ricorrenti, nonché personali interpretazioni della realtà vissuta, al fine di estrapolare alcune istanze pedagogiche sottese. Come sostiene Bakhtin (1981), i processi narrativi richiedono un'interazione e un ascolto dialogico (dialogical relationships), allo scopo di portare alla luce la complessità della ricerca narrativa. Il discorso invita a considerare l'intreccio di almeno tre voci: la voce del narratore; la cornice teorica, che fornisce i concetti e gli strumenti per l'interpretazione; un monitoraggio riflessivo dell'atto della lettura e dell'interpretazione da parte del ricercatore (Lieblich, TuvalMashiach, et al., 1998).

È da considerare come lo scrivente abbia seguito il flusso di pensieri e di idee che si è andato a dipanare nel discorso e, al contempo, mediante un processo abduttivo, abbia intrecciato gli stessi con contributi teorici di riferimento.

Si è consapevoli della non esaustività della trattazione e dei limiti intrinseci di un'analisi basata su quanto spontaneamente emerso dalle narrazioni. I medesimi aspetti sono meritevoli di ulteriori sviluppi e approfondimenti. Altresì, si ritiene possano aiutare a indagare in via esplorativa alcune dimensioni significative per lo sviluppo della compagine familiare. L'approccio narrativo ha permesso di osservare come la pandemia e le relative conseguenze abbiano talvolta ridestato vissuti legati ai primi tempi di vita insieme o a situazioni particolarmente critiche attraversate da genitori e figli. Inoltre, molte delle competenze sviluppate nel percorso adottivo hanno costituito per le famiglie utili strumenti per far fronte con flessibilità e duttilità alla situazione in atto. 


\section{Avvio della famiglia adottiva e similitudini con la vicenda emergenziale}

Parlare di adozione significa affrontare aspetti quali precarietà, vulnerabilità, incertezza, imprevedibilità, ma anche flessibilità, resilienza, risorse. Fin dalle sue fasi iniziali, l'avvio della famiglia adottiva si connota come una «svolta cruciale», un «evento critico» (Rosnati, Ranieri, et al., 2003, p. 25) contrassegnato da un carico di stress per genitori e figli (Brodzinsky, Pinderhughes, 2002).

Altresì, la formazione e la preparazione si rivelano fattori protettivi per una riorganizzazione e rimodulazione dei tempi e dei ritmi del nucleo domestico. In prospettiva sistemico-relazionale, tale compito coinvolge in modo differente i membri del sistema familiare e l'ambiente circostante (Pati, 2014). Nel ciclo di vita familiare si coglie un dinamismo di reciprocità fra genitori e figli, in una continua negoziazione del patto adottivo (Scabini, Cigoli, 2000), e un vicendevole scambio interattivo tra famiglia e mondo esterno.

In prospettiva educativa, si procederà portando all'attenzione gli aspetti evidenziati, allo scopo di rintracciare competenze e buone prassi per affrontare la precarietà dell'attuale panorama mondiale.

\subsection{Abitare l'incertezza}

Nell'iter adottivo non esiste un periodo certo di gestazione, come potrebbero essere i nove mesi della gravidanza. Neppure è sicuro che il desiderio si traduca in una concreta possibilità di adozione. Di norma, l'adozione segue un'attesa più o meno lunga, segnata da momenti di scoramento, difficoltà, percorsi di valutazione. Genitori e figli attraversano una sorta di limbo nel quale, pur nella fatica, diviene opportuno rinvenire risorse e strumenti utili a fronteggiare agli imprevisti che irrompono nel cammino. Non tutte le coppie giungono a guardare a questo tempo sospeso come a un'opportunità: talvolta, accade di essere sopraffatti dalle criticità incontrate e di vivere tutto in funzione del giorno che verrà. Anche la transizione alla genitorialità adottiva avviene con uno scarso preavviso, che implica una veloce riorganizzazione domestica e non sempre è accordato il giusto tempo per congedarsi e chiudere alcune pratiche personali e lavorative.

Capacità di accettazione e flessibilità adattiva di genitori e figli si palesano come due potenziali fattori protettivi nel processo adottivo (Rosnati, 1998). Si coglie il valore della ricerca di una continuità nella discon- 
tinuità e di imparare ad abitare l'imprevisto (Paradiso, 2018; Di Silvio, 2013). Le medesime competenze paiono rivestire un ruolo decisivo nelle famiglie a seguito della diffusione su larga scala di SARS-CoV-2. Nel nostro Paese, l'emergenza epidemiologica ha comportato prese di decisioni impreviste e improvvise, implicando una ridefinizione dei ritmi di vita familiari. Una madre adottiva, al riguardo, afferma: «non potendo controllare la situazione, ho accettato la situazione e cercato di vivere serenamente»; un'altra persona mette in luce come si siano rese necessarie «nuove strategie di vita familiare e sociale».

$\mathrm{Ci}$ si può interrogare su come il non poter fare progetti a lungo termine e il so-stare nell'incertezza siano riconducibili a quanto vissuto da genitori e figli durante l'attesa. L'aver vissuto un periodo nel quale non era possibile avere certezze e serviva essere aperti alle novità o agli ostacoli che si intercalavano nell'iter adottivo sembra costituire un babitus per i genitori adottivi. Si ricupera la disponibilità a "vivere alla giornata", ricercando il senso dischiuso tra le pieghe del quotidiano.

Aver esperito simili eventi non significa, necessariamente, affrontare quanto si ripresenta nell'oggi con lo stesso atteggiamento. È richiesto un precipuo lavoro su di sé e un'accettazione incondizionata della realtà. Dalle narrazioni si coglie come l'evento pandemico, una situazione per eccellenza imprevista e inimmaginabile agli occhi umani, susciti paura, rabbia e senso impotenza. Il desiderio sotteso alle parole dei partecipanti è che "presto tutto abbia fine" e si possa tornare all'ordinarietà della vita quotidiana. C'è chi riporta un forte senso di ingiustizia, in particolare rispetto alle privazioni che ne sono derivate per i figli, e chi sembra attestarsi ad un piano di "adattamento", nell'ottica di un più ampio disegno di tutela sociale.

I genitori in questione, pur nelle peculiarità delle esperienze vissute, paiono aver fatto proprio uno stile di analisi delle situazioni che va oltre il limite esperito e si volge al possibile che ancora non è visibile. Si rileva la disponibilità a compiere un adeguato esame di realtà, accettando quanto sconvolge i progetti personali, pur con spirito critico e propositivo, e volgendosi a inedite mete future. Questo stile permette di so-stare nell'incertezza rinvenendo le risorse presenti.

\subsection{Conciliazione famiglia-lavoro}

In relazione all'ambito di impiego, si pensi ai professionisti sociosanitari o alle forze dell'ordine, per alcuni il periodo dell'emergenza è 
coinciso con ritmi di lavoro più serrati, preoccupazione sul rischio di contagio e minor tempo a disposizione per le cure familiari. Due genitori affermano di essere stati «molto appesantiti», in quanto impegnati in turni di lavoro complementari (la madre lavorava di giorno e il padre di notte), pensati apposta per conciliare l'accudimento del figlio. Gli stessi hanno rilevato un'evidente «difficoltà e stanchezza nella gestione del bambino, senza poter contare su un sostegno esterno alla famiglia». Una madre sostiene che è stato «pesante passare dodici ore da sola con il bambino» mentre il marito era fuori casa per il lavoro.

La situazione venutasi a creare con il lockdown ha reso più difficile trovare spazi personali, senza essere fagocitati dai compiti di cura. Questo aspetto riconduce alla nascita della famiglia adottiva e a quel periodo in cui, almeno uno dei genitori, è impegnato a tempo pieno nell'accudimento. I ricordi che riaffiorano sono pregni di emozioni e di opportunità; altresì, non è negata la fatica sul piano psico-fisico e nella gestione degli episodi di conflittualità. Gli adulti rischiano di incorrere in momenti di scoramento e si rende indispensabile il supporto dell'altro coniuge e di una rete relazionale e/o professionale di sostegno.

A causa del Covid-19, le famiglie hanno dovuto ripensare, nell' hinc et nunc, i propri ritmi di vita e le consuetudini. Il protrarsi dello stato di emergenza ha richiesto un differente pensiero circa la conciliazione dei tempi lavorativi e familiari.

La pandemia non ha reso possibile una riorganizzazione graduale. Il quadro di complessità è accresciuto dalla necessità di combinare presso il proprio domicilio, l'espletamento dell'attività lavorativa e l'assunzione dei compiti di cura verso i propri membri (minori, persone disabili o non autosufficienti, anziani; Centro Studi di Ateneo, a cura di, 2020, cfr. in particolare le pp. 17-18).

Sono in particolare i lavoratori con bambini piccoli a esser stati interessati da un ingente lavoro in ambito professionale così come nella cura, finanche ad «eccedere le loro capacità di resistenza» (Centro di Ateneo Studi e Ricerche sulla famiglia, a cura di, 2020, p. 57). Il non poter contare sull'appoggio dei servizi o dei nonni ha significato per qualcuno un periodo di congedo dal lavoro, l'opzione di lavorare in smart-working o di telelavoro, l'inserimento di figure di supporto nella cura dei figli. Come ribadisce un genitore adottivo, questo equilibrio giunge non senza fatica, sia in relazione «al doversi adattare al nuovo modo di lavorare sia per la gestione dell'elaborazione delle emozioni del figlio, oltre che all'accompagnamento nel percorso scolastico». Si è così andata a delineare una situazione dai labili confini tra famiglia e lavoro, nella quale 
il tempo del lavoro può prevaricare e invadere il tempo delle relazioni familiari o viceversa. Una madre, al riguardo, confida: «purtroppo, dovendo lavorare, mi sono spesso innervosita nel vedere che nostro figlio non rispettava mai la piccola regola di non disturbarmi se ero al telefono per lavoro e la mia frustrazione ogni tanto ha avuto la meglio».

In un tempo colmo di sfide e di criticità, i genitori adottivi ritengono che, per far fronte con maggiore efficacia alle sfide affrontate, sia indispensabile ridefinire alcune priorità; rinegoziare i tempi e gli spazi di vita familiare; disporsi all'ascolto dei bisogni altrui, alla loro comprensione e alla ricerca di comuni vie alternative di cambiamento.

Anche in questo caso, l'esperienza offre una base di partenza, un'attitudine, ma non elimina la fatica di riprogettare nell'oggi la vita familiare e di ricercare il continuo equilibrio tra vita lavorativa e familiare. Una madre, al riguardo, afferma: «ho vissuto, da un lato, con nervosismo questo momento, dovuto in parte al lavoro, in parte alla didattica a distanza, e all'impossibilità di uscire per nostro figlio; dall'altro con la serena accettazione di un momento in cui si stava in casa e si passava il tempo insieme nel miglior modo possibile». Accanto alle fatiche e alle preoccupazioni, si intravede la possibilità di "recuperare tempo" con i figli e il partner.

\subsection{Gestione dello stress}

Possiamo rilevare come l'epidemia planetaria da SARS-CoV-2 abbia rappresentato una minaccia e un evento stressante per la vita individuale e sociale. I timori per la salute personale e dei propri cari, le preoccupazioni economiche e lavorative, le limitazioni delle frequentazioni, la difficile conciliazione fra lavoro e famiglia, un diffuso senso di smarrimento e incertezza hanno minato il procedere di molte famiglie (Centro Studi di Ateneo, 2020, p. 15). La situazione di stress varia in relazione ai singoli nuclei domestici e alla fase del ciclo di vita familiare attraversata.

La pandemia ha sconvolto ritmi e routine consolidate, con conseguenze sul piano delle emozioni e degli agìti. Una madre confida che i momenti di frustrazione del figlio «sono stati molto più marcati, pianti continui e nervosismo per la scuola online. Inoltre, sono stati frequenti i capricci per qualsiasi cosa e sono aumentati gli attacchi di rabbia fisici, da contenere». Non di rado, ciò richiede un ulteriore lavoro in ambiti nei quali, in precedenza, la famiglia aveva già raggiunto un discreto equilibrio. Un'altra madre si sofferma, al riguardo, sulla fatica di dover "ripartire da zero" e del riproporsi di criticità verificatesi nei primi mesi di convivenza. Lad- 
dove, i minori presentano particolari bisogni educativi o una diagnosi di disabilità l'assenza di continuità nel supporto specialistico o terapeutico acuisce il disagio e comporta regressioni e involuzioni nel percorso.

L'emergenza ha ridestato, sovente, traumi passati. Il lockdown, in alcuni minori adottati, ha evocato ricordi legati all'istituzionalizzazione e alle separazioni significative vissute. Un genitore sostiene che la chiusura fra le mura domestiche ha sollecitato nel figlio il pensiero del tempo trascorso in istituto e delle limitazioni vissute in quel contesto.

Un altro bambino è tornato a riproporre paure latenti: «paura della morte e bisogno di continue rassicurazioni». In questo caso, si è reso indispensabile «un supporto psicologico per aiutarlo a superare timori e paure ricorrenti».

Ai genitori compete favorire una risignificazione degli eventi, mediante l'apertura al dialogo e al confronto sulla memoria adottiva. Una madre rileva come il figlio abbia spesso «parlato - più per fantasia che per ricordi - del proprio Paese di origine»; un' altra confida come il figlio «si sia soffermato maggiormente su domande inerenti alla propria storia personale».

Si tratta di occasioni preziose per mantenere aperta la comunicazione sull'adozione: openess in adoption (Wrobel, Kohler, et al., 2003). Un percorso progressivo, che inizia fin dai primi anni di vita del bambino con una storia semplice e comprensibile ed evolve con lo sviluppo del minore in relazione alle sue esigenze e al grado di maturità raggiunto (Brodzinsky, Pinderhughes, 2002). Ogni situazione di crisi e di emergenza mina la sicurezza personale, induce timori e ritrosie, chiede la capacità di abitare con coraggio l'imprevisto e di cogliere i significati sottesi dietro le pieghe del vissuto.

Dall'esperienza dei genitori adottivi si rileva una attitudine a "vigilare" sul percorso di crescita dei figli, sui momenti di crisi e sulle fatiche che si presentano nel cammino, mettendo in campo competenze possedute o ricercando un sostegno mirato laddove necessario. Al centro del discorso, si attesta l'esigenza di maturare nelle relazioni familiari inedite capacità di decentramento da sé, di comprensione dei vissuti, di comunicazione educativa (Simeone, 2008).

\subsection{Distanziamento sociale tra rafforzamento dei legami familiari e isolamento}

Qualche coppia ha rivisitato nella situazione pandemica la fatica dei primi tempi insieme, dove si affrontano sfide inedite in solitudine. Le fa- 
miglie adottive sono accumunate da un periodo iniziale di distanziamento sociale: un salto temporale e geografico durante il quale accordare priorità alla costruzione dei legami familiari.

Nell'adozione internazionale, l'incontro e i primi tempi insieme si svolgono concretamente in uno spazio altro per genitori e figli, distante da quello che sarà l'avvio della convivenza nel nuovo Paese di accoglienza. Non di rado, nel Paese estero, può accadere che, per questioni di sicurezza, siano vietati gli spostamenti al di fuori dell'abitazione se non accompagnati. Anche nell'adozione nazionale, i genitori incontrano il figlio nel contesto di accoglienza ed è previsto un percorso progressivo di conoscenza.

In fase di avvio, è necessario un periodo dedicato per "fare famiglia". Sono essenziali momenti condivisi fra genitori e figli, nei quali conoscersi, instaurare una relazione educativa, definire routine familiari. Attraverso la via del dialogo, del gioco, dell'affettività vengono poste le fondamenta per la condivisione, il riconoscimento reciproco e la costruzione di una comune appartenenza. Ciò può comportare il ripensamento delle proprie frequentazioni e dei ritmi di vita, ponendo al centro i bisogni di sicurezza e di appartenenza del figlio. Questo implica anche la disponibilità a procrastinare un eventuale inserimento del minore in contesti di socialità, quali la scuola o i servizi educativi $i^{3}$ Al contempo, è essenziale il supporto ricevuto "a distanza" da parte di altre famiglie adottive o professionisti che offrono ascolto e incoraggiamento.

Il quadro presentato porta con sé un duplice livello di analisi. Nell'adozione, il distanziamento sociale è accostabile da un lato, alla necessità di rinsaldare i legami familiari e consentire al figlio il giusto tempo per fidarsi e ambientarsi; dall'altro lato, al rischio di chiusura e solitudine che, in particolare, i genitori sperimentano nel rapido mutamento di abitudini, nell'assunzione di una funzione educativa che mina le sicurezze precedentemente costruite, nella limitazione delle frequentazioni. Si rendono necessari una postura personale, un fare spazio dentro sé al minore, una ridefinizione delle priorità ed alcune rinunce in vista dell'accoglienza del figlio, pur nella ricerca di contesti di supporto personali o di coppia. Il distanziamento sociale non è pertanto sinonimo di autoreferenzialità e isolamento.

${ }^{3}$ Le linee di indirizzo per il Diritto allo Studio degli alunni adottati prevedono la possibilità di ritardare l'ingresso a scuola e di concordare con le Istituzioni tempi consoni con il percorso di ciascun minore (MIUR, 2014). 
I partecipanti alle serate online, nella maggior parte dei casi, hanno inteso il lockdown come esperienza privilegiata per accrescere i legami familiari, così come accaduto al momento dell'avvicinamento, quando per un periodo ci si è dedicati "solo al figlio". Un'occasione per trascorrere più tempo insieme, rallentare i ritmi, avere «molto tempo a disposizione, meno stress e riscoprire alcuni momenti insieme».

In particolare, il telelavoro ha ampliato le possibilità di incontro con i familiari. Un padre sottolinea come il periodo in cui erano entrambi a casa sia stato una risorsa per approfondire il rapporto con la figlia; un'altra madre afferma che «per certi aspetti è stato molto piacevole: abituati a non avere il papà a casa per tutta la giornata e avere la possibilità di averlo maggiormente presente è stato bello per tutti».

Al contempo, non di rado, l'isolamento si traduce in solitudine e chiusura. Il lockdown ha segnato in modo profondo la socialità e la formazione delle giovani generazioni. Un padre adottivo con rammarico espone la situazione del figlio per il quale, negli anni, avevano tessuto «una rete relazionale e di supporto anche professionale, in rispondenza ai suoi bisogni speciali». Dall'oggi al domani è venuto meno ogni tipo di intervento, se non un minimo appoggio a distanza da parte dell'insegnante di sostegno che, tuttavia, non si è rivelato funzionale alle esigenze del minore. La Didattica a Distanza non ha garantito a tutti le medesime opportunità e ha, talvolta, acuito le disuguaglianze, la povertà educativa e il rischio di dispersione scolastica (Save the Children, 2020).

I genitori, inoltre, nella conciliazione tra tempi della famiglia e del lavoro, non possono più contare sul prezioso supporto di servizi e reti relazionali. L'isolamento presso il proprio domicilio comporta l'interruzione della frequentazione di qualsiasi contesto socio-educativo, scolastico ed extrascolastico, così come il sostegno sociale offerto da parenti e/o amici. È possibile cogliere le ripercussioni per un minore adottato che abbisogna di alimentare le relazioni sociali e familiari con continuità e stabilità. Si pensi anche, in molti casi, ai disagi dovuti ad un contesto abitativo angusto o all'impossibilità di giocare all'aria aperta e di trascorrere del tempo fuori dalla propria abitazione. Una mamma così si esprime: «è mancato il non poter uscire e fare attività a contatto con la natura».

Infine, urge riflettere su come non sempre la vicinanza fisica sia sinonimo di benessere familiare. Si fa riferimento a quanti hanno attraversato una condizione di forte precarietà lavorativa o hanno perso il lavoro in seguito alle ripercussioni della pandemia sul sistema economico. Le preoccupazioni impediscono di vivere con serenità il quotidiano. 
Non va poi trascurato come il Covid-19 abbia causato lutti inattesi, negando al morente la vicinanza dei suoi cari e ai familiari un ultimo congedo. Questo risulta ancora più difficile da elaborare per un minore adottato, con alle spalle un passato di separazioni e cesure. Una madre adottiva narra come nella cerchia familiare, a causa del nuovo coronavirus, si siano verificati alcuni decessi improvvisi e sia stato complesso accompagnare il figlio a comprendere questo distacco in assenza di riti concreti e senza poter contare sul conforto e sostegno di presenze amiche.

Disorientamento, sofferenza, solitudine hanno minato l'integrità personale e causato smarrimento e disagio diffuso. Il concetto di vulnerabilità rinvia alla finitudine e alla fragilità costitutive dell'esperienza umana, posizionandosi in un continuum incerto e precario tra ben-essere e mal-essere. In un tempo segnato dalla pandemia si assiste al venir meno di quell'ambiente supportivo che può accompagnare a riscoprire le risorse possedute e a far fronte alle sfide attraversate ${ }^{4}$. Nel periodo dell'emergenza sanitaria, i genitori in questione hanno espresso gradimento verso il percorso on-line promosso dall'associazione, in quanto ha permesso di confrontarsi; rilevare strategie e buone prassi introdotte da altri genitori; «non sentirsi soli»; «affrontare tematiche utili rispetto alla situazione vissuta».

\subsection{Politiche familiari}

Dedicare tempo a "fare famiglia" richiede la revisione delle politiche familiari. Nel caso dell'adozione, non sempre i tempi richiesti per l'ambientamento e l'incontro tra genitori e figli si conciliano con opportune misure e politiche del lavoro. Il congedo parentale è concesso solo ad uno dei genitori, nell'adozione nazionale in seguito a un decreto di affidamento provvisorio, mentre nell'adozione internazionale può essere parzialmente goduto prima dell'ingresso del minore in Italia. Altresì, è possibile contare su un'aspettativa non retribuita, né indennizzata, nel periodo di permanenza all'estero'.

${ }^{4}$ In relazione al ciclo di vita attraversato e alla vulnerabilità dei singoli nuclei domestici (Milani, Ius, et al., 2013), gli studi mostrano talvolta un acuirsi della conflittualità intra-familiare, finanche a giungere a situazioni di grave rischio per adulti e minori. A titolo esemplificativo, si rimanda agli episodi di violenza agita o assistita tra le mura domestiche; al crescente numero di vittime di genere o di suicidi al termine del lockdown (Polizia di Stato, 2020).

${ }^{5}$ D.lgs. 23 marzo 2001, n. 151, Testo unico sulla maternità e paternità, art. 26, co. 4. 
È palese come tutto ciò non sia sufficiente nel percorso adottivo, in quanto durante l'avvicinamento, che in Italia può risolversi nel lasso di qualche settimana, mentre all'estero può protrarsi fino ad alcuni mesi, è richiesta la presenza continuativa di entrambi i genitori. La legge non riconosce appieno il diritto dei genitori di assentarsi dal lavoro per dedicarsi alla costruzione della nuova famiglia e, tale situazione, è ancora fortemente soggetta alla discrezionalità del datore di lavoro o ad un periodo di astensione dal lavoro non retribuito. A questo, nell'adozione internazionale, si aggiungono le spese da sostenere per la pratica adottiva, il viaggio e la permanenza nel Paese estero. Si rende urgente un più ampio sistema di politiche di conciliazione, quali per esempio un ripensamento del congedo parentale, riconosciuto al contempo alla figura paterna e materna, o una flessibilità lavorativa agevolmente fruibile dai lavoratori.

È opportuno riflettere su come tali misure debbano essere accessibili anche a convivenza avviata. Ogni bambino o ragazzo affronta il cambiamento in modo inedito. Tuttavia, nell'adozione ciò è acuito dal fatto che esso richiama i sentimenti abbandonici alla base delle esperienze traumatiche precoci vissute. Di fronte al presentarsi di imprevisti o eventi che comportano la separazione dalle figure di riferimento o l'inserimento in nuovi contesti sociali, il minore abbisogna di adeguato sostegno e del tempo necessario per ambientarsi.

L'esperienza pandemica ha messo in luce la fragilità del sistema di politiche educative familiari. Alcuni genitori rivelano di essersi sentiti «dimenticati e lasciati soli ad affrontare le criticità connesse con la conciliazione fra famiglia e lavoro». Per far fronte all'emergenza in atto sono state introdotte, talvolta con ritardo rispetto al bisogno, forme di flessibilità (es. congedi parentali, lavoro agile), misure economiche di sostegno ai genitori lavoratori, fondi straordinari per l'assistenza a familiari non autosufficienti o con disabilità, sostegni fiscali (es. versamenti sospesi, soppressione delle clausole di salvaguardia in materia di IVA e accise, tax credit vacanze, ecobonus), protezione dei redditi (es. sospensione delle procedure di licenziamento, cassa integrazione in deroga, indennità) ${ }^{6}$.

$\mathrm{Al}$ riguardo, è auspicabile prospettare un ampio disegno di politiche sociali che, alle provvidenze concrete affianchi percorsi di avvaloramento della famiglia, secondo una logica promozionale e partecipativa. Que-

${ }^{6}$ Per ulteriori approfondimenti, si veda quanto introdotto dal Governo a partire dal Decreto "Cura Italia", sul sito ministeriale https://www.mef.gov.it (data di ultima consultazione: 30.1 .21 ). 
sto implica un ripensamento dei bisogni delle famiglie e la garanzia di un equo sostegno a quanti si trovano a barcamenarsi nel difficile equilibrio tra famiglia e lavoro.

\subsection{Fiducia nelle Istituzioni}

L'iter adottivo si dipana all'interno di un percorso istituzionale nel quale professionisti e Autorità preposte si adoperano per il buon esito del processo, assicurando un'idonea formazione e valutazione degli aspiranti genitori adottivi e un'adeguata preparazione del minore all'adozione.

Serve piena fiducia nelle Istituzioni, anche laddove non è facile comprendere i significati sottesi ad alcune decisioni. Gli aspiranti genitori, consegnando la propria disponibilità all'adozione, co-partecipano al percorso adottivo, pur nella consapevolezza di non poter controllare tutto e di dover dipendere dalla valutazione dei professionisti coinvolti e dalle decisioni dell'Autorità competente. Ci sono tempi di attesa che si accettano con fatica, situazioni che comportano un alto carico di tensione, fattori di contesto socio-politico o procedurale che appaiono poco corrispondenti al diritto del minore alla famiglia. Si rileva, non di rado, un senso di impotenza e frustrazione in adulti e minori che interpretano il ruolo dell'Autorità competente come un'ingerenza nella sfera di vita personale. Agli stessi è chiesto di transitare da una motivazione all'adozione incentrata sul progetto familiare coniugale, all'accesso al significato sociale dell'adozione (Paradiso, 2015).

Anche rispetto all'emergenza sanitaria da Covid-19, ai cittadini è stato chiesto di attenersi a quanto definito dall'Autorità in materia di sicurezza e prevenzione. L'eccezionalità della situazione e la difficoltà di comprendere la realtà dei fatti hanno suscitato timori e disagio emotivo. Le restringenti misure di protezione e le limitazioni imposte sono, talvolta, state vissute con frustrazione, rabbia e senso di impotenza.

Tali eventi non attesi e non desiderati portano a sperimentare il limite. Dalle narrazioni dei genitori adottivi si rintracciano stili personali di coping rispetto alle implicazioni della pandemia nella sfera di vita personale. Alcuni genitori appaiono ancorati al tentativo di far emergere il malessere dei figli e delle famiglie, in relazione alle limitazioni disposte e la chiusura delle scuole e dei servizi educativi. Altri, invece, partono descrivendo una realtà quasi idilliaca, mettendo in luce le potenzialità dischiuse nella convivenza domestica e, solo nel prosieguo del discorso, 
si soffermano sulle criticità esperite. A metà strada, si collocano coloro che, pur desiderando ricercare strategie per riconsegnare ai figli occasioni di socializzazione e apprendimento al di fuori delle mura domestiche, sembrano optare per la messa in atto di azioni che possano trasformare in occasione l'emergenza in corso.

L'ultimo atteggiamento appena descritto racchiude in sé una via utile al potenziamento della resilienza familiare. Secondo Le Breton, il limite «risponde a una necessità antropologica» (1991, trad. it. 1995, p. 19), in quanto consente alla persona di raggiungere una più approfondita conoscenza di sé, imparare «a riconoscersi, a distinguersi dagli altri, a restaurare un valore alla sua esistenza» (ivi, pp. 19-20). Entrare in contatto e accogliere la precarietà e la vulnerabilità diviene il primo passo per maturare consapevolezza e volgersi verso orizzonti inediti. Ne deriva l'urgenza di un continuo equilibrio fra accettazione del dato di realtà e protagonismo, al fine di evitare di incorrere in un duplice rischio: da un lato, quello di inseguire mete irraggiungibili e non alla propria portata; dall'altro, quello di subire scelte e decisioni in maniera acritica. Si tratta di sviluppare percorsi di empowerment familiare, incrementando la capacità di analizzare le situazioni, di attivarsi autonomamente e responsabilmente, di accettare quanto non si può modificare, di governare il cambiamento.

\section{Accrescere la resilienza}

La resilienza, intesa quale capacità di far fronte agli eventi avversi e traumatici dell'esistenza, fa leva sulle risorse possedute o disponibili nell'ambiente di riferimento (Cyrulnik, 1999, trad. it. 2000). Secondo Cyrulnik, essa «non è un elenco di qualità possedute da un individuo, ma un processo che, dalla nascita alla morte, l'individuo intreccia continuamente con il suo ambiente» (2001, trad. it. 2002, p. 219). In quanto processo, viene alimentata in modo dinamico nel corso dello sviluppo e nell'intersezione fra fattori individuali, familiari ed ambientali (Garmezy, 1985). Il contesto di vita in cui il soggetto è inserito, infatti, può incoraggiare o inibire la resilienza, così come costituire un fattore protettivo o di rischio.

Con Walsh possiamo parlare di resilienza familiare come «il modo in cui una famiglia affronta e gestisce un'esperienza perturbante, contiene i livelli di stress, si riorganizza in maniera adeguata e prosegue la sua vita» (1998, trad. it. 2008, p. 19). 
Le famiglie adottive che si è avuto modo di ascoltare si mostrano come famiglie resilienti. La duttilità e la capacità di stare nella precarietà, uno sguardo attento all'altro da sé, la riprogettazione del quotidiano, l'assunzione delle proprie responsabilità genitoriali costituiscono i presupposti per una tenuta pur nelle situazioni critiche che si presentano.

Fin dal loro nascere, le famiglie adottive sono sollecitate a fare proprie flessibilità e duttilità per affrontare le incertezze e le sfide dell'esperienza adottiva. Le medesime si confrontano con situazioni di precarietà e sono chiamate a sviluppare personali strategie di coping. Gli studi mettono in luce come il pre-adozione sia segnato da situazioni di forte stress e tensioni (Macario, 2010). Altresì, l'avvio della convivenza si connota come un evento critico nel ciclo della famiglia adottiva, carico di emotività per adulti e minori (Paradiso, 2015). I membri del sistema domestico coltivano nel tempo questa disponibilità a adattarsi alle vicende vissute, avendo come meta il benessere della compagine familiare.

Si coglie come i genitori adottivi appaiono sensibili al vissuto e ai bisogni dei figli, in particolare, in relazione alle ripercussioni delle esperienze sfavorevoli pregresse sulla crescita. Le peculiarità dell'esperienza adottiva richiedono infatti uno sguardo attento, vigile e paziente e costante dialogo fra genitori e figli. L'empatia e l'accoglienza percepita dal minore da parte delle figure adulte di riferimento sono alla base della costruzione della memoria adottiva e del senso del sé e del noi familiare (Paradiso, 2018).

Altresì, le medesime attenzioni rappresentano uno stimolo ad assumersi piena responsabilità nel compito di educare ed istruire i figli, ricercando con creatività occasioni ed esperienze quotidiane arricchenti e di corresponsabilità fra scuola e famiglia. Il lockdown ha richiesto ai genitori di occuparsi di dimensioni talvolta demandate alle Istituzioni educative. Tale passaggio, se colto come opportunità, dispiega un inedito potenziale per la crescita delle relazioni familiari e per riscoprirsi interlocutori privilegiati nel rapporto con le Istituzioni.

Preme, infine, riflettere su come non tutto possa essere risolto fra le mura domestiche. La famiglia è chiamata ad alimentare gli scambi con l'ambiente circostante e a giovarsi del contributo che proviene dalla comunità locale. In questo, l'esperienza associativa offre sostegno e preziose occasioni formative e di condivisione.

Alla luce delle sfide poste dalla pandemia, è opportuno soffermarsi sulla valenza di percorsi formativi che incoraggino processi di riflessività e sollecitino la persona a «riconsiderare la propria esperienza pratica come fattore d'apprendimento continuo, a riorganizzare il proprio 
rapporto con il contesto esperienziale, ad assegnare nuovi significati all'ambiente in cui si trova e al proprio agire» (Pati, 2013). I medesimi percorsi, facendo leva sulle potenzialità possedute, devono incoraggiare processi trasformativi ed empowerment familiare (Simeone, 2008). Emerge il valore di coltivare un dialogo interiore: un ascolto di sé che aiuti a so-stare nella situazione attraversata, accrescere la consapevolezza, rinvenire strategie di azione, affinare la comprensione empatica.

\section{Conclusioni}

Nel percorso della famiglia adottiva l'incertezza è compagna di viaggio in ogni fase del cammino. Genitori e figli si trovano ad affrontare l'emergenza, la precarietà, la difficoltà di gestire l'imprevisto, l'assenza di elementi oggettivi circa la storia di vita che ha preceduto l'avvio della convivenza, la sofferenza connessa con le esperienze sfavorevoli pregresse. Di fronte a tali eventi, la flessibilità e la riflessività si rivelano preziose alleate.

La prima porta con sé una disposizione a adattarsi alle situazioni e a ricercare inediti approcci alla realtà. In questo modo, è possibile mettere in questione il proprio progetto di vita familiare e renderlo più consono a quanto esperito, pur nella fedeltà ai valori posti a fondamento della vita coniugale.

La seconda è da intendersi quale processo che si dispiega in relazione al contenuto di un problema; alle modalità di risoluzione dello stesso; alle premesse da cui si parte per affrontare la questione (Mezirow, 2003). Secondo Dewey, per essere genuinamente pensanti, le persone devono «sostenere e protrarre quello stato di dubbio che stimola ad una completa ricerca, in modo da non accettare un'idea o asserire positivamente una credenza finché non si siano trovate fondate ragioni per giustificarla» (2010, trad. it. 1973, p. 77). La riflessività si delinea pertanto come componente di un più ampio processo di apprendimento intenzionale $\mathrm{e}$ trasformativo.

Ogni famiglia, di fronte all'emergenza sanitaria da Covid-19 è stata chiamata a riconsiderare il proprio funzionamento e i ritmi di vita. Le risorse e le strategie emerse dai racconti appaiono uno stimolo per ripensare alla conciliazione fra tempi di vita familiare e lavorativa, in vista del perfezionamento dei membri del nucleo domestico.

Al contempo, l'attenzione verso i bisogni e le esigenze dei figli, porta a comprendere l'urgenza di interrogarsi circa i vissuti dei minori rispet- 
to a questa situazione emergenziale. Il senso di smarrimento, fragilità e incertezza che mina le nuove generazioni si è acuito nel corso dell'anno trascorso. Fra i giovani, sono in via di emersione inediti fattori di rischio, disagio e devianza aggravati dalla situazione di distanziamento sociale, dalle limitazioni indotte e dalla difficoltà di rinvenire aiuti dal mondo adulto per risignificare l'esistenza e la sofferenza attraversata.

La riflessione compiuta non può esulare da un riferimento conclusivo ai minori e alle coppie ancora in attesa di adozione. Se il periodo del lockdown ha segnato una momentanea interruzione delle adozio$\mathrm{ni}$ in ambito nazionale ed internazionale, i tempi di ripresa risultano ancora critici a livello internazionale. Le limitazioni e i blocchi sulla mobilità, nonché i timori circa i contagi, rendono molto prudenti gli Stati esteri rispetto all'autorizzazione a procedere con l'adozione internazionale.

L'auspicio è che un precipuo lavoro tra gli Enti autorizzati, le Autorità dei Paesi, le Ambasciate Italiane e la Commissione Adozioni Internazionali nel nostro Paese, possa continuare a garantire le corrette misure per assicurare continuità e portare a conclusione gli iter adottivi avviati.

\section{Riferimenti bibliografici}

Bakhtin M.M. (1981): The Dialogic Imagination. Four Essay. Austin (TX): University of Texas Press.

Brodzinsky D.M., Pinderhughes E.E. (2002): Parenting and Child Development in Adoptive Families. In M.H. Bornstein (eds.): Handbook of Parenting. Vol I. Children and Parenting. Mahawah (NJ): Lawrence Erlbaum Associates.

Centro di Ateneo Studi e Ricerche sulla famiglia (a cura di) (2020): La famiglia sospesa. Milano: Vita e Pensiero.

Cohen N.J., Coyne J., Duvall J. (1996): Parents' Sense of “entitlement" in Adoptive and Nonadoptive Families. Family Processes, n. 35, pp. 441-456.

Cyrulnik B. (1999): Il dolore meraviglioso. Trad. it. Milano: Frassinelli, 2000.

Cyrulnik B. (2001): I brutti anatroccoli. Le paure che ci aintano a crescere. Trad. it. Milano: Frassinelli, 2002.

Dewey J. (1910): Come pensiamo: una riformulazione del rapporto tra il pensiero e l'educazione. Trad. it. Scandicci (Fi): La Nuova Italia, 1973.

Di Silvio R. (2013): Qualcuno che ha la mia stessa faccia. In R. Pregliasco (a cura di): Alla ricerca delle proprie origini. L'accesso alle informazioni tra norma e cultura. Roma: Carocci.

Garmezy N. (1985): Stress-Resistant Children: The Search for Protective Factors. In J.E. Stevenson (ed.): Recent Research in Developmental Psychopathology. Oxford: Pergamon Press. 
Gunner M.R., Kertes D.A. (2005): Rischi prenatali e postnatali nello sviluppo neurobiologico dei bambini nelle adozioni internazionali. In D.M. Brodzinsky, J. Palacios (a cura di): Lavorare nell'adozione. Dalle ricerche alla prassi operativa. Trad. it. Milano: FrancoAngeli, 2011.

Istat (2020): Fase 1: Le giornate in casa durante il lockdown. 5 aprile - 21 aprile 2020. (https://www.istat.it/files//2020/06/Giornate_in_casa_durante_ lockdown.pdf; data di ultima consultazione: 4.2.21).

Le Breton D. (1991): Passione del rischio. Trad. it. Torino: Gruppo Abele, 1995.

Lieblich A., Tuval-Mashiach R., Zilber T. (1998): Narrative Research: Reading, Analysis and Interpretation. London: Sage.

Macario G. (2010): I tempi dell'attesa: una sfida formativa per la qualità. In Commissione per le Adozioni Internazionali (a cura di): La qualità dell'attesa nell'adozione internazionale. Significati, percorsi, servizi. Firenze: Istituto degli Innocenti.

Mezirow J. (1991): Apprendimento e trasformazione. Il significato dell' esperienza e il valore della riflessione nell'apprendimento degli adulti. Trad. it. Milano: Raffaello Cortina, 2003.

Milani P., Ius. M., Serbati S. (2013): Vulnerabilità e resilienza: lessico minimo. Studium Educationis, n. 3, pp. 72-80.

MIUR (2014): Linee di indirizzo per favorire il diritto allo studio degli alunni adottati (https://www.istruzione.it/allegati/2014/prot7443_14_all1.pdf; data di ultima consultazione: 4.2.21).

Newton Verrier N. (1993): La ferita primaria. Comprendere il bambino adottato. Trad. it. Milano: Il saggiatore, 2007.

Paradiso L. (2015): Prepararsi all'adozione. Le informazioni, le leggi, il percorso formativo personale e di coppia per adottare un bambino. Nuova edizione. Milano: Unicopli.

Paradiso L. (2018): Narrazioni familiari e adozione. Il ruolo degli operatori, insegnanti e genitori. Francavilla al Mare (Ch): Edizioni Psiconline.

Pati L. (1999): Adozione internazionale e sua dimensione educativa. In Id. (a cura di): L'educazione familiare alla prova: adottare un bambino straniero. Riflessioni pedagogiche sull' adozione internazionale. Milano: I.S.U. - Università Cattolica.

Pati L. (2013): Formazione dei genitori ed esigenze organizzative. La Famiglia, n. 257, pp. 23-29.

Pati L. (2014): Il divenire sistemico della famiglia fra tempo e spazio educativi. In Id. (a cura di): Pedagogia della famiglia. Brescia: La Scuola.

Pati L. (2017): Editoriale. La Famiglia, n. 261, pp. 5-9.

Polizia di Stato (2020): ...Questo non è amore. Italia (https://www.poliziadistato.it/statics/28/2020-impag-1_32-opuscolo-polizia-definitivo.pdf; data di ultima consultazione: 30.1 .21 ).

Rosnati R. (1998): La costruzione del legame adottivo: un'impresa congiunta di genitori e figli. In D. Bramanti, R. Rosnati (a cura di): Il patto adottivo. L'adozione internazionale di fronte alle sfide dell'adolescenza. Milano: Franco Angeli. 
Rosnati R., Ranieri S., Greco O. (2003): La costruzione della genitorialità adottiva. In O. Greco, S. Ranieri, R. Rosnati (a cura di): Il percorso della famiglia adottiva. Strumenti per l'ascolto e l'accompagnamento. Milano: Unicopli.

Save the Children (a cura di) (2020): Riscriviamo il Futuro. L'impatto del coronavirus sulla povertà educativa. Italia (https://s3.savethechildren.it/public/ files/uploads/pubblicazioni/limpatto-del-coronavirus-sulla-poverta-educativa_0.pdf; data di ultima consultazione: 30.1.21).

Scabini E., Cigoli V. (2000): Il famigliare. Legami, simboli e transizioni. Milano: Raffaello Cortina.

Simeone D. (2008): Educare in famiglia. Indicazioni pedagogiche per lo sviluppo dell'empowerment familiare. Brescia: La Scuola.

Walsh F. (1998): La resilienza familiare. Trad. it. Milano: Raffaello Cortina, 2008.

Wrobel G.M., Kohler J.K., Grotevant H.D., McRoy R.G. (2003): The Family Adoption Communication Model (FAC): Identifying Pathways of Adoption-Related Communication. Adoption Quarterly, n. 7, pp. 53-84. 